J. F. Reichardt - J. W. Goethe Briefwechsel

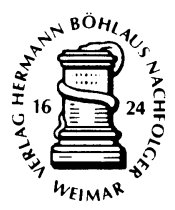




\section{J.F. Reichardt - J.W. Goethe Briefwechsel}

herausgegeben und kommentiert von

Volkmar Braunbehrens, Gabriele Busch-Salmen, Walter Salmen 
J. F. Reichardt - J. W. Goethe : Briefwechsel /

hrsg. und kommentiert von Volkmar Brauenbehrens ...

- Weimar : Verl. Hermann Böhlaus Nchf., 2002

ISBN 978-3-7400-1194-9

ISBN 978-3-476-02856-3 (eBook)

DOI 10.1007/978-3-476-02856-3

Dieses Werk einschließlich aller seiner Teile ist urheberrechtlich geschützt. Jede Verwertung außerhalb der engen Grenzen des Urheberrechtsgesetzes ist ohne Zustimmung des Verlages unzulässig und strafbar. Das gilt insbesondere für Vervielfältigungen, Übersetzungen, Mikroverfilmungen und die Einspeicherung und Verarbeitung in elektronischen Systemen.

www.boehlausnf.de info@böhlausnf.de 


\section{Inhalt}

Vorwort

Einleitungen

Gabriele Busch-Salmen:

Die Briefe Reichardts an Goethe

Volkmar Braunbehrens:

Die Briefe Goethes an Reichardt

Briefwechsel

Kommentar

Anhang

Die Familie Reichardt

Begegnungen zwischen Goethe und Reichardt ...... 227

Register der erhaltenen und verschollenen Briefe .. 228

Verzeichnis der Siglen und Abkürzungen ............... $\quad 230$

Personenregister

233

Bildnachweis 


\section{Vorwort}

Schon in der Konzeption vieler Werke von Goethe spielt die Musik eine wichtige Rolle. Ein großer Teil seiner Lyrik sollte als "Lieder" verstanden sein, im "Wilhelm Meister"-Roman spielen die eingestreuten "Gesänge " eine herausragende Rolle und große Teile seiner dramatischen Werke wurden als "Singspiele" oder "Schauspiele mit Gesang" ausgearbeitet und bezogen die Musik (und den Tanz) als wesentliches gestalterisches Mittel ein. Obschon Goethe weniger ein schöpferischproduktives als ein von ausgeprägten Vorstellungen geleitetes rezeptives Verhältnis zur Musik hatte, wird man doch von einem integralen Anteil der Musik an seinem Werk sprechen müssen, der bislang wenig beachtet worden ist. (Das obwohl besonders die Komponisten - seit Goethes Zeiten bis heute - das geradezu zur Musik drängende Potential in Goethes Dichtkunst erkannt und Texte von ihm in einem Ausmaß wie kaum bei einem anderen Dichter zur Grundlage ihrer Musik gewählt, sie in Musik gesetzt, vertont oder zur musikalischen Gestaltung bearbeitet haben.)

Für Goethe war das Zusammenwirken mit Komponisten, die auf seine musikalischen Vorstellungen eingingen und die entsprechenden Werke erst zu einem "Ganzen " auszubilden halfen, unverzichtbar. Zunächst war es der Jugendfreund aus Frankfurter Tagen, Philipp Christoph Kayser, mit dem Goethe bis etwa 1789 eng zusammen arbeitete und der ihm nicht nur zu grundlegenden Kenntnissen zur Musikgeschichte verhalf, sondern als anregender und kenntnisreicher Gesprächspartner seine immer deutlicher werdenden Vorstellungen von Singspiel und Oper begleitete. In Goethes Alterszeit wurde Carl Friedrich Zelter zu einem der engsten und vertrautesten Freunde, der einerseits Goethes Dichtungen nach dessen Intentionen in Musik setzte, andererseits Goethes Urteil über die musikalischen Produktionen der jüngeren Zeitgenossen 
wesentlich beeinflußte, aber auch entscheidende Anstöße gab zu Goethes theoretischen Reflexionen zu einer allgemeinen "Tonlehre", die allerdings zu keinem abgeschlossenen Werk mehr führten.

In der Zeit nach dem Abbruch der unbefriedigend und persönlich schwierig gewordenen Beziehung zu Kayser (1789) bis zum (1797 begonnenen, aber erst ab 1802 intensiveren) Freundschaftsverhältnis zu Carl Friedrich Zelter wurde der Berliner Hofkapellmeister Johann Friedrich Reichardt zum wichtigsten musikalischen Partner Goethes, der nicht nur Goethesche Singspiele in Musik setzte, sondern auch etwa 140 Goethe-Lieder, Balladen, "Deklamationsstücke", und Musik zu Goethes Schauspielen (wie z. B. "Egmont«) in einer für den Dichter mustergültigen Weise komponierte.

Die Bedeutung dieser Reichardtschen Goethe-Kompositionen, - gerade weil sie sich so eng an Goethes Vorstellung vom Verhältnis von Text und Musik orientierten und seine eigenen Intentionen verwirklichten, - aber auch die Dokumentation einer sowohl für die Goethe-Biographie wie auch für die Reichardt-Forschung überaus gewichtigen, in seinen Höhen und Tiefen problematischen und dramatischen $\mathrm{Zu}$ sammenarbeit, geben dem Briefwechsel zwischen Goethe und Reichardt ein besonderes Gewicht. Er erscheint anläßlich von Reichardts 250. Geburtstag am 25. November 2002.

Johann Friedrich Reichardt (1752-1814) war nicht nur als Berliner Hofkapellmeister und Komponist tätig, sondern auch als Musikhistoriker, Zeitschriftenherausgeber, Verleger, Reiseschriftsteller und belletristischer Autor, darüber hinaus in vielfältiger Weise auch zu musikalischen Fragen und im Gebiet der politischen Publizistik. Sein umfangreicher Briefwechsel mit Vertretern all dieser Interessengebiete, den er zudem mit zahlreichen bedeutenden Dichtern wie Klopstock, Claudius, Herder, Hamann, Ludwig Achim von Arnim, Tieck und anderen führte, ist, wenn er auch nur zu Teilen erhalten ist, zu wenig beachtet worden. Denn er beleuchtet eine von der Aufklärung bis zur frühen Romantik reichende kulturelle Umbruchphase ebenso wie die politischen Veränderungen von der Zeit Friedrichs II. über die französische Revolution 
bis zu den "Befreiungskriegen" von der Napoleonischen Herrschaft. In all dem war Reichardt als ein heftig beteiligter, um deutliche Worte nie verlegener Zeitgenosse engagiert. Wegen seiner widerspruchsvollen Biographie und seines unsteten und manchmal aufdringlich erscheinenden Charakters bot er allerdings viele Angriffsflächen und galt als eine der umstrittenen Persönlichkeiten seiner Zeit. Auch das Verhältnis zu Goethe litt unter solchen persönlichen und politischen Belastungen, so daß Goethe später rückblickend von Reichardt sagte, er sei "von der musikalischen Seite unser Freund, von der politischen unser Widersacher".

In der vorliegenden Ausgabe des Briefwechsels zwischen Goethe und Reichardt werden alle noch vorhandenen oder überlieferten Briefe in chronologischer Folge mitgeteilt, - die nicht erhaltenen, aber durch sekundäre Belege erschließbaren in einem Briefverzeichnis erfaßt. Goethes Briefe werden hier nach der sog. Sophienausgabe (WA), jedoch - soweit vorhanden - nach den Handschriften überprüft mitgeteilt.

Kurz vor der Drucklegung dieses Bandes konnte der Bestand an Briefen, die Goethe an Reichardt adressierte, durch die Wiederauffindung des Schreibens vom 18. Oktober 1789 komplettiert werden. Die Universitäts- und Landesbibliothek Münster besitzt seit 1983 einen Teil des Nachlasses des mit Reichardt verwandten Historikers Kurt von Raumer, unter dessen reichem Briefbestand ein wohl vor der Entnahme des Autographs am 3. Juli 1942 angefertigtes Faksimile des Goethebriefes zu finden war. Über den Verbleib des Autographs ist nichts bekannt.

Die Briefe Reichardts werden nach den im Goethe- und Schiller-Archiv in Weimar befindlichen Handschriften wiedergegeben, die teilweise erheblich von der Ausgabe durch Max Hecker im Goethe-Jahrbuch von 1925 abweichen. Die den Briefen, insbesondere von Reichardt oft beigefügten Beilagen (Briefe anderer Absender, Notenmanuskripte, Notenausgaben, Bücher) sind in den meisten Fällen nicht mehr vorhanden und werden diesfalls in den Kommentaren, soweit eruierbar, aufgeführt. Die Kommentare zu den Briefen Goethes schrieb Volkmar Braunbehrens, die zu Reichardts Briefen Walter Salmen. 
Da die Beziehung von Reichardt und Goethe zueinander kompliziert, durch äußere Einflüsse belastet, plötzlich abgebrochen und erst nach Jahren wieder aufgenommen, schließlich unerklärt beendet wurde, schien es den Herausgebern zweckmäßig, auch die in den Briefen nicht deutlich ausgetragenen Konflikte und Vorbehalte zum besseren Verständnis in zwei sich ergänzenden, die verschiedenen Perspektiven berücksichtigenden Einleitungen vorzustellen. Gelegentliche Überschneidungen und wiederkehrende Zitate schienen uns dabei ein leicht verzeihliches Übel. Gabriele Busch-Salmen beleuchtet in ihrem Text Goethes Bedeutung für Reichardt auf dem Hintergrund einer widersprüchlichen Biographie, Volkmar Braunbehrens zeigt Goethes Interesse an einer künstlerischen Partnerschaft und sein Verhalten, wo diese gefährdet schien.

Als gemeinsame Autoren dieser Briefedition möchten wir uns gegenseitig für die überaus förderliche und angenehme interdisziplinäre Zusammenarbeit bedanken, die sowohl literaturhistorische als auch musikhistorische Fragen zu einer kongenialen musikalischen Partnerschaft Reichardts mit Goethe zur Diskussion stellen möchte.

Die Herausgeber 